\title{
Low-intensity focused ultrasound (LIFU)-induced acoustic droplet vaporization in phase-transition perfluoropentane nanodroplets modified by folate for ultrasound molecular imaging
}

\author{
This article was published in the following Dove Press journal: \\ International Journal of Nanomedicine \\ 27 January 2017 \\ Number of times this article has been viewed
}

Jianxin Liu',*
Tingting Shangl,*
Fengjuan Wang'
Yang Cao'
Lan Hao'
JianLi Ren ${ }^{1,2}$
Haitao Ran ${ }^{1,2}$
Zhigang Wangl,2
Pan Li,2
Zhiyu Du
'Chongqing Key Laboratory of
Ultrasound Molecular Imaging,
${ }^{2}$ Department of Ultrasound,
${ }^{3}$ Postgraduate Department, The
Second Affiliated Hospital of
Chongqing Medical University,
Chongqing, People's Republic of China
*These authors contributed equally
to this work

Correspondence: Zhiyu Du; Pan Li Chongqing Key Laboratory of Ultrasound Molecular Imaging, The Second Affiliated Hospital of Chongqing Medical University, Linjiang Road 76, Chongqing, 4000I0,

People's Republic of China

Tel +8623 637| 96I2

Fax +8623637 I 1527

Email dr.duzhiyu@163.com; cqlipan@163.com

\begin{abstract}
The commonly used ultrasound (US) molecular probes, such as targeted microbubbles and perfluorocarbon emulsions, present a number of inherent problems including the conflict between US visualization and particle penetration. This study describes the successful fabrication of phase changeable folate-targeted perfluoropentane nanodroplets (termed FA-NDs), a novel US molecular probe for tumor molecular imaging with US. Notably, these FA-NDs can be triggered by low-intensity focused US (LIFU) sonication, providing excellent US enhancement in B-mode and contrast-enhanced US mode in vitro. After intravenous administration into nude mice bearing SKOV3 ovarian carcinomas, 1,1'-dioctadecyl-3,3,3',3' -tetramethylindotricarbocyanine iodide-labeled FA-NDs were found to accumulate in the tumor region. FA-NDs injection followed by LIFU sonication exhibited remarkable US contrast enhancement in the tumor region. In conclusion, combining our elaborately developed FA-NDs with LIFU sonication provides a potential protocol for US molecular imaging in folate receptor-overexpressing tumors.

Keywords: low-intensity focused ultrasound, perfluoropentane nanodroplets, acoustic droplet vaporization, ultrasound molecular imaging, targeting
\end{abstract}

\section{Introduction}

Ultrasound (US) molecular imaging has made remarkable progress over the last decade and has been attracting attention due to the unique advantages of ultrasonography including real-time observation, non-invasiveness, free of radiation, relatively low cost, and portability. ${ }^{1-4}$ Targeted microbubbles (MBs) have been the most prevalent form of US molecular probes, which have been used to diagnose tumor, inflammatory lesions, atherosclerotic plaque, and thrombi. The applications of molecular US have been expanded to targeting therapy since MBs could serve as drug carriers for local release in combination with US-targeted MB destruction, which provides a novel and powerful tool for tumor targeting imaging, therapy, monitoring, and treatment evaluation. ${ }^{5-7}$ This technology has great potential for future clinical applications; however, MBs as US molecular probes and drug carriers are challenged by several inherent problems. First, the micro-size of MBs does not allow effective extravasation into tumor tissue, even though it has defective vasculature with much bigger capillary endothelium gaps (range, 380-780 nm) compared to normal tissues. ${ }^{8}$ This limits molecular US modality to intravascular imaging and therapy, which indicates that direct targeting of MBs to tumor cells is not possible. Unfortunately, few specific angiogenic markers 

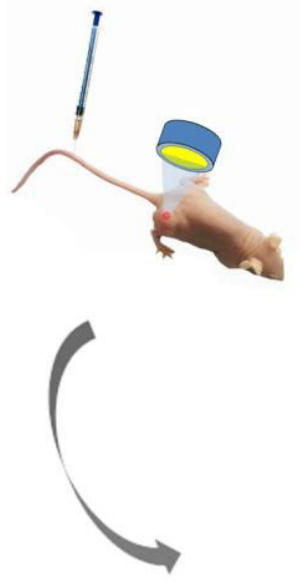
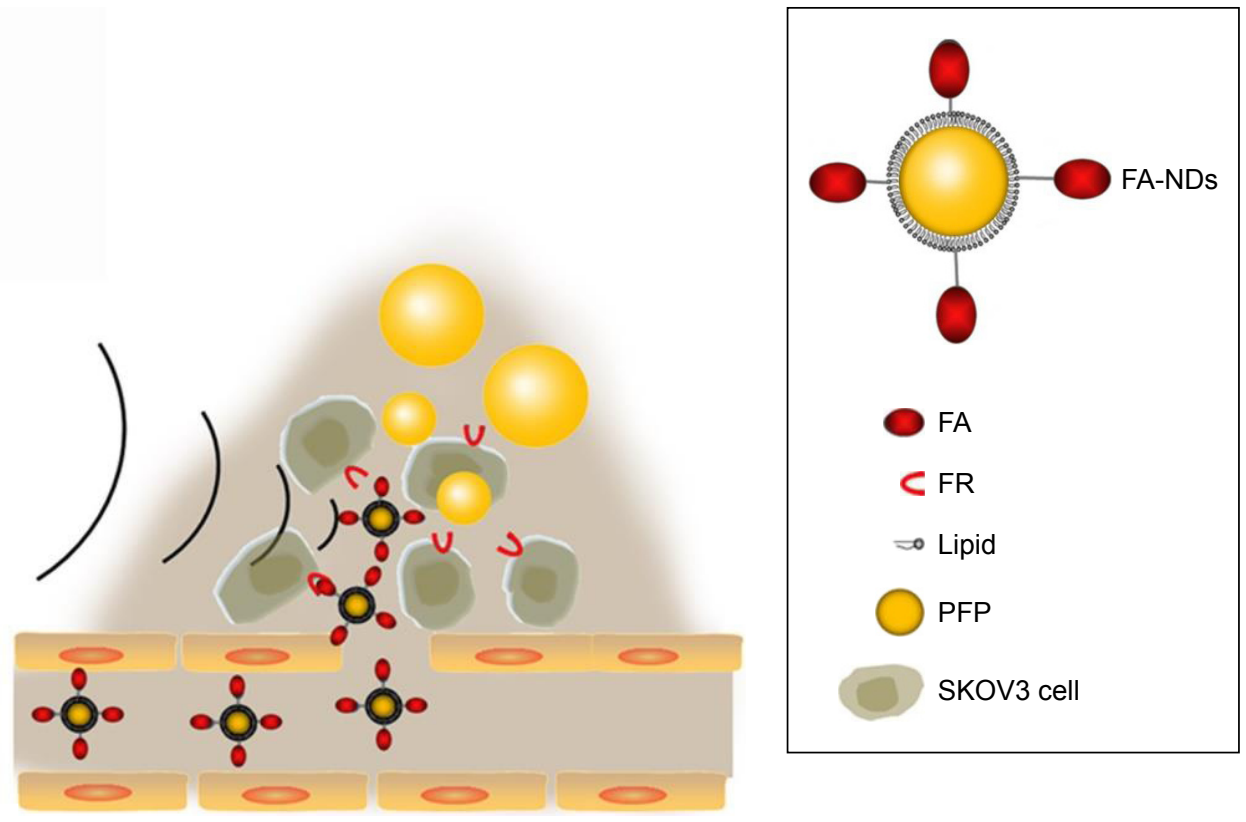

Figure I Schematic illustration of FA-ND extravasation into tumor tissue.

Note: The diagram depicts FA-ND microstructure and the phase-transition process of FA-NDs after LIFU sonication.

Abbreviations: FA-ND, folate-targeted perfluoropentane nanodroplet; LIFU, low-intensity focused ultrasound; FR, folate receptor; PFP, perfluoropentane.

are overexpressed on tumor vascular endothelial cells. This significantly narrows down the diagnostic and therapeutic options of molecular US..$^{910}$ Moreover, the very short circulation time of MBs (half-life of 3-15 min) prevents effective targeting imaging and $\mathrm{MB}$ destruction for tumor-specific drug release. All these disadvantages seriously hamper the application of MB-based US molecular imaging and therapy.

To overcome the limitation of MBs as molecular probes, several types of liquid perfluorocarbon (PFC) emulsion nanodroplets were developed for US molecular imaging; their small size enables extravasation into tumor tissue and fair stability to facilitate long circulation time in vivo. ${ }^{11,12}$ Nevertheless, PFC nanodroplets cannot effectively enhance US imaging in vivo as gas-filled MBs did because of their poor echogenicity. ${ }^{13}$ To solve the conflict between US imaging and particle penetration, phase-transition technology was introduced by developing nanoscaled MB precursors that effectively accumulate in tumor tissue by passive or active targeting and then convert into MBs in situ under tumordirected US. ${ }^{14,15}$ Notably, our groups recently fabricated PFC nanosystems capable of phase transition that successfully delivered to release drugs and affect gene expression, ${ }^{16}$ facilitate multimodal imaging, ${ }^{17,18}$ and increase the therapeutic efficiencies of high-intensity focused US (HIFU) and chemoembolization. ${ }^{19}$ The liquid-gas phase transition of perfluorohexane ( $\mathrm{PFH}$, boiling point $[\mathrm{BP}] 58^{\circ} \mathrm{C}-60^{\circ} \mathrm{C}$ ) can be triggered by hyperthermia under HIFU; however, this operation is risky and invasive and does not allow intraoperative imaging. ${ }^{19}$

This study developed phase-changeable and folate receptor (FR)-targeted nanodroplets with a low BP $\left(29^{\circ} \mathrm{C}\right) \mathrm{PFC}$ (perfluoropentane, PFP) core (termed FA-NDs) for use as a novel US molecular probe that can pass through the capillary endothelium gaps of tumor blood vessels and actively target FR-positive tumor cells. Importantly, different from prior work to activate phase transition with HIFU, this is the first time PFC nanodroplet phase transition has been induced by low-intensity focused US (LIFU). The facile phase-convertible PFP core provides the advantage of preventing damage to the surrounding healthy tissues. Folate, a highly stable and non-immunogenic small molecule, was conjugated to the nanodroplet surface to further increase its tumor targeting ability. ${ }^{20,21}$ The targeting efficiency and phase-transition effect under heating or LIFU irradiation for US imaging were simultaneously investigated in vitro and in vivo. FA-ND extravasation into tumor tissue, the phase-transition process of FA-NDs after LIFU irradiation, and FA-ND microstructure are depicted in Figure 1.

\section{Materials and methods Materials}

Lipid compounds including 1-palmitoyl-2-stearoyl-snglycero-3-phosphocholine (HSPC), 1,2-distearoylsn-glycero-3-phosphoethanolamine-N-[amino 
(polyethyleneglycol)-2000] [DSPE-PEG(2000)], 1,2distearoyl-sn-glycero-3-phosphoethanolamine-N[amino(polyethyleneglycol)-2000]-folate [DSPE-PEG (2000)-folate], 1,2-dipalmitoyl-sn-glycero-3-phospho(1'-rac-glycerol) (DPPG), and cholesterol were obtained from Avanti Polar Lipids, Inc. (Alabaster, AL, USA). Free folate; PFP $\left(29^{\circ} \mathrm{C} \mathrm{BP}\right)$; and fluorescent dyes including 4',6-diamidino-2-phenylindole (DAPI), 1,1'-dioctadecyl$3,3,3^{\prime}, 3^{\prime}$-tetramethylindotricarbocyanine iodide (DiR), 1,1'-dioctadecyl-3,3,3',3'-tetramethylindocarbocyanine perchlorate (DiI), and 3,3'-dioctadecyloxacarbocyanine perchlorate $(\mathrm{DiO})$ were purchased from Sigma-Aldrich Chemical Co. (St Louis, MO, USA). Chloroform $\left(\mathrm{CHCl}_{3}\right)$ was purchased from Fisher Scientific (Waltham, MA, USA).

\section{Preparation and characterization of FA-ND}

Lipid compounds were mixed together [HSPC, DSPEPEG (2000)-folate, DPPG and cholesterol in a molar ratio of 10:4:3:3, $20 \mathrm{mg}$ of total amount] and then dissolved in $10 \mathrm{~mL}$ chloroform, which was then removed with a rotary vacuum evaporator (Yarong Inc, Shanghai, People's Republic of China). Two hours later, the resulting thin lipid films were hydrated in $4 \mathrm{~mL}$ phosphate-buffered saline (PBS, pH 7.4). After adding $200 \mu \mathrm{L}$ of PFP, the suspension was emulsified in an ice bath using a sonicator (Sonics \& Materials Inc., Newtown, CT, USA) with power of $130 \mathrm{~W}$ for $5 \mathrm{~min}$ ( $5 \mathrm{~s}$ on and $5 \mathrm{~s}$ off), then the folate-targeted PFP nanoemulsions were harvested. To remove free lipids and excess reactants, the nanoemulsions were centrifuged at 7,500 rpm for $5 \mathrm{~min}$ and then washed in PBS. The centrifugation and washing process were repeated three times. Finally, the prepared FA-NDs were stored at $4^{\circ} \mathrm{C}$ for later use. The preparation of non-targeted control PFP nanodroplets (termed non-NDs) were prepared as above except that DSPE-PEG (2000)-folate was replaced with DSPE-PEG (2000) at the same molar ratio. To prepare fluorescent nanodroplets, DiI (1 mg) or DiR (1 mg) fluorescent dye was added to the lipid solution with silver papers to prevent light exposure.

Particle distribution and morphological characterization were determined with optical microscopy (CKX41; Olympus, Tokyo, Japan) and confocal laser scanning microscopy (CLSM) (A1R; Nikon, Tokyo, Japan). FA-ND structure was analyzed with a transmission electron microscope (TEM) (H-7600; Hitachi, Tokyo, Japan). The mean particle size and zeta potential were determined by dynamic light scattering (Malvern Instruments, Malvern, UK). PFP nanoemulsions were stored at $4{ }^{\circ} \mathrm{C}$, and the mean particle size was measured at different time points after preparation $(6,12,18,24,30$, $36,42,48,54,60,66$, and $72 \mathrm{~h}$ ).

\section{FA-ND thermal evaporation}

PFP nanoemulsions ( $1 \mathrm{mg} / \mathrm{mL}$ of FA-NDs) of $10 \mu \mathrm{L}$ were placed in a hot plate (Biotherm, Inc., Beijing, People's Republic of China) and temperature was adjusted to $40^{\circ} \mathrm{C}$, $45^{\circ} \mathrm{C}, 50^{\circ} \mathrm{C}$, and $55^{\circ} \mathrm{C}$. PFP nanoemulsions in the hot plate were observed with an optical microscope, and the images of nanodroplets were recorded before and after heating. Other PFP nanoemulsions ( $1 \mathrm{mg} / \mathrm{mL}$ of FA-NDs, $1 \mathrm{~mL}$ ) in plastic balloons were placed in a water bath kettle (YarongInc) to observe the US image of nanoemulsions after evaporation from water suspension. The water temperature was set at $40^{\circ} \mathrm{C}, 45^{\circ} \mathrm{C}, 50^{\circ} \mathrm{C}$, and $55^{\circ} \mathrm{C}$. Equivalent degassed water in balloons were used as controls. The US imaging was performed and recorded in B-mode and contrast-enhanced US (CEUS) mode using an US system (Esaote MyLab90, Florence, Italy) with frequency of $5-12 \mathrm{MHz}$ and mechanical index (MI) of 0.06 . The echo intensity of the region of interest was determined by using US imaging analysis software (Chongqing Medical University, Chongqing, People's Republic of China). ${ }^{22}$

\section{US imaging of nanodroplets after acoustic droplet vaporization (ADV)}

An LIFU instrument ${ }^{23}$ (LM.SC051 ACA; Institute of Ultrasound Imaging of Chongqing Medical Sciences, Chongqing, People's Republic of China) with a driving frequency of $1.0 \mathrm{MHz}$, focal length of $1.5 \mathrm{~cm}$, focus area of $0.4 \mathrm{~cm}^{2}$, acoustic intensity in a focal spot of $0.4-3.2 \mathrm{~W} / \mathrm{cm}^{2}$, and $50 \%$ duty cycle was used in this study in a pulse wave (PW) mode with different acoustic intensities $(0.8,1.6,2.4$, and $3.2 \mathrm{~W} / \mathrm{cm}^{2}$ ) for $2 \mathrm{~min}$. LIFU acoustic intensity was measured with a hydrophone (HNA-0400; ONDA Corp., Sunnyvale, CA, USA). After $200 \mu \mathrm{L}$ of FA-ND nanoemulsions $(1 \mathrm{mg} / \mathrm{mL})$ were placed in a gel mold ( $3 \%$ agar w/v in distilled water), the LIFU instrument was placed perpendicular to the surface of the gel mold to elicit ADV of PFP nanodroplets. A degassed water-filled sac was placed between the LIFU probe and gel mold to ensure that the focus of LIFU sonication was in the center of the nanoemulsion. Imaging of nanoemulsions was obtained with the US system, and the echo intensities of the regions of interest were determined by US imaging analysis software. Finally, the liquid in the gel mold was sucked out for optical microscopy to observe whether any MBs had been produced. 


\section{Cell culture}

The SKOV3 human ovarian cancer cell line, which was obtained from Chongqing Key Laboratory of Ultrasound Molecular Imaging (Chongqing, People's Republic of China), was selected for this research because it overexpresses the FR. The cells were cultured in RPMI-1640 medium supplemented with $10 \%$ fetal bovine serum, $100 \mathrm{U} / \mathrm{mL}$ penicillin, and $100 \mathrm{U} / \mathrm{mL}$ streptomycin. The cells were maintained in an incubator with a humidified atmosphere containing $5 \%$ $\mathrm{CO}_{2}$ at $37^{\circ} \mathrm{C}$. Cells in a logarithmic growth phase were used for the experiments. All cell culture reagents were purchased from Invitrogen (Carlsbad, CA, USA).

\section{In vitro FA-ND targeting}

SKOV3 cells seeded in culture dishes specified for CLSM at a density of $1 \times 10^{4}$ cells per dish were divided into three groups. After $\sim 24 \mathrm{~h}$, the cells formed a stable monolayer at the bottom of each dish, and then different treatments were applied to different groups. The folate-targeted group was treated with $100 \mu \mathrm{L}$ DiI-labeled FA-NDs nanoemulsions $(1 \mathrm{mg} / \mathrm{mL})$. The non-targeted group was supplemented with $100 \mu \mathrm{L}$ DiIlabeled non-NDs nanoemulsions $(1 \mathrm{mg} / \mathrm{mL})$. The antagonism group was incubated with free FA $(1 \mathrm{~mol} / \mathrm{L}, 1 \mathrm{~mL})$ for $1 \mathrm{~h}$ and then mixed with $100 \mu \mathrm{L}$ DiI-labeled FA-NDs nanoemulsions (1 $\mathrm{mg} / \mathrm{mL})$. After a 45-min co-incubation with nanoemulsions, the cells in all three groups were washed with PBS three times, fixed with $4 \%$ paraformaldehyde $(1 \mathrm{~mL})$ for $20 \mathrm{~min}$, and incubated with $\mathrm{DiO}(1 \mu \mathrm{g} / \mathrm{mL}, 200 \mu \mathrm{L})$ and DAPI $(1 \mu \mathrm{g} / \mathrm{mL}, 200 \mu \mathrm{L})$ for $20 \mathrm{~min}$ each. After paraformaldehyde and each fluorescent dye administration, dishes were washed with PBS three times. Finally, the dishes were wrapped in silver paper to avoid light and sent for CLSM.

\section{Animal culture}

Female immunodeficient BALB/c nude mice (6-8 weeks, $18-24 \mathrm{~g}$ ) were bred and housed between $19^{\circ} \mathrm{C}$ and $22^{\circ} \mathrm{C}$ and humidity conditions. Food and water were provided ad libitum. All the experimental protocols were approved by the Institutional Animal Care and Use Committee of Chongqing Medical University. The experimental operations were carried out in accordance with the protocol approved by the Institutional Animal Care and Use Committee of Chongqing Medical University. To induce solid tumors, $1 \times 10^{6}$ cells in $100 \mu \mathrm{L}$ PBS ( $\mathrm{pH}=7.4$ ) were subcutaneously injected into the right flank of every mouse.

\section{In vivo targeting efficiency}

Twenty days after tumor inoculation, 12 mice bearing xenograft SKOV3 tumors (10 cm in diameter) were randomly divided into two groups (targeted and non-targeted groups) for fluorescence imaging (FLI). All the mice were anesthetized with intraperitoneal injections of $1 \%$ sodium pentobarbital $(6.25 \mathrm{~mL} / \mathrm{kg})$, and then $200 \mu \mathrm{L}$ dilutions of FA-ND or non-ND nanoemulsions stained with DiR were injected via the tail vein. The PFP dose relative to the body weight of a mouse was $100 \mu \mathrm{L} / \mathrm{kg}$, which was lower than the dose $(126 \mu \mathrm{L} / \mathrm{kg})$ reported to result in no side effects except mild pulmonary hyperinflation. ${ }^{24}$ Then the mice were observed using an FLI system (CRi Inc, Woburn, MA, USA) at 0.5, 1,6 , and $12 \mathrm{~h}$ postinjection with excitation and emission wavelengths of 748 and $780 \mathrm{~nm}$, respectively. The tumorto-background ratio of fluorescence intensity, which was calculated by dividing the mean fluorescence intensity of the tumor region by that of the surrounding tissue, was recorded $0.5,1,6$, and $12 \mathrm{~h}$ after intravenous nanoemulsion administration. Tissues including brain, heart, lung, liver, spleen, kidney, and tumor nodes were completely extracted for additional ex vivo FLI $12 \mathrm{~h}$ after injection, and the mean fluorescence intensities were recorded. Sixteen separate mice bearing xenograft SKOV3 tumors $(10 \mathrm{~cm}$ in diameter) were randomly divided into two groups (targeted and non-targeted groups) for fluorescence microscopy of tumor tissues. The mice received the same treatment as above except that the nanoemulsions were stained with DiI. At 0.5, 1,6 , and $12 \mathrm{~h}$ postinjection, tumors were sent for ultrathin sectioning $(6 \mu \mathrm{m})$ immediately after extraction from nude mice and fluorescent microscopy processing and imaging (CKX41; Olympus).

\section{In vivo ADV and US imaging}

Three weeks after tumor inoculation, 18 mice bearing SKOV3 tumors ( $10 \mathrm{~cm}$ in diameter) were randomly divided into three groups $(n=6)$ and subjected to FA-ND nanoemulsion injection followed by LIFU sonication, non-ND nanoemulsion injection followed by LIFU sonication or FA-ND injection. All the mice received a $200-\mu \mathrm{L}$ nanoemulsion injection via tail vein (100 $\mu \mathrm{L} / \mathrm{kg}$ body weight). LIFU sonication ( $2 \mathrm{~min}$ ) was performed $1 \mathrm{~h}$ after nanoemulsion injection in $\mathrm{PW}$ mode with acoustic intensity of $3.2 \mathrm{~W} / \mathrm{cm}^{2}$. A degassed water-filled sac was placed between the LIFU probe and the tumor surface to ensure that the focus was in the tumor. An increase in the echo intensity value, which was calculated by subtracting the postinjection tumor echo intensity from the preinjection value, was recorded after observation.

\section{Statistical analysis}

Statistical Package for the Social Sciences software (SPSS; IBM, Armonk, NY, USA) was used for statistical analyses. 
All data are presented as mean \pm standard deviation. One-way analysis of variance was utilized for statistical evaluation, and differences were considered statistically significant at $P<0.05$.

\section{Results}

\section{Nanodroplet characterization}

FA-ND nanoemulsions appeared as uniformly milky suspensions after harvest. Nanodroplets were punctiform in shape under an optical microscope and a CLSM (Figure 2A and B) and showed spherical morphology on TEM imaging (Figure 2C). The mean diameter of the prepared nanodroplets was $321 \pm 67 \mathrm{~nm}$ with a polydispersity index of 0.430 (Figure 2D), and the average surface zeta potential was $-50 \mathrm{mV}$ (Figure 2E). The mean diameter and average surface zeta potential of non-NDs were $315 \pm 62 \mathrm{~nm}$ and $-53 \mathrm{mV}$, respectively. There was no significant difference between FA-ND and non-ND nanoemulsions in terms of mean particle size and zeta potential. The mean particle size of FA-ND nanoemulsions did not vary significantly within $48 \mathrm{~h}$ at a storage temperature of $4^{\circ} \mathrm{C}$ (Figure 2F). After 2 days, the nanoemulsions would stratify and convert to a milky suspension after gentle shaking, with slightly enlarged particle size.

\section{In vitro evaporation of PFP nanoemulsions}

At $40^{\circ} \mathrm{C}$ in the hot plate, the FA-NDs showed no obvious changes, and almost no MBs were observed under the microscope (Figure 3A). Many nanodroplets enlarged to form MBs when the temperature rose to $45^{\circ} \mathrm{C}$ (Figure 3B). Almost all nanodroplets enlarged/fused and abundant MBs formed when the temperature reached $50^{\circ} \mathrm{C}$ (Figure 3C). Only a few larger bubbles could be observed (Figure 3D) at $55^{\circ} \mathrm{C}$. These microscopic observations were consistent with ultrasonography results of nanoemulsions in water suspensions, which showed nearly no contrast-enhanced signals acquired at $40^{\circ} \mathrm{C}$ (Figure 3E). Moderate, strong, and minimal contrast enhancement was evident at $45^{\circ} \mathrm{C}$ (Figure $3 \mathrm{~F}$ ), $50^{\circ} \mathrm{C}$ (Figure $3 \mathrm{G}$ ), and $55^{\circ} \mathrm{C}$ (Figure $3 \mathrm{H}$ ), respectively. The quantitative results of echo intensity measured with US imaging analysis software were also in accordance with US imaging findings (Figure 3I and J).

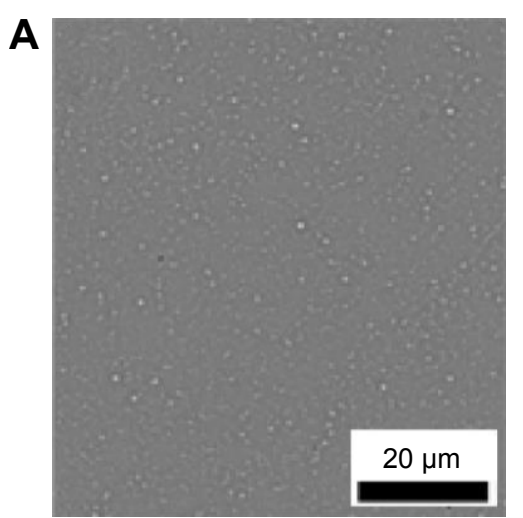

D

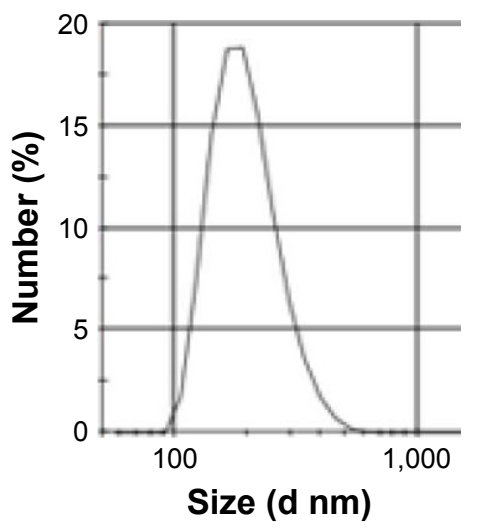

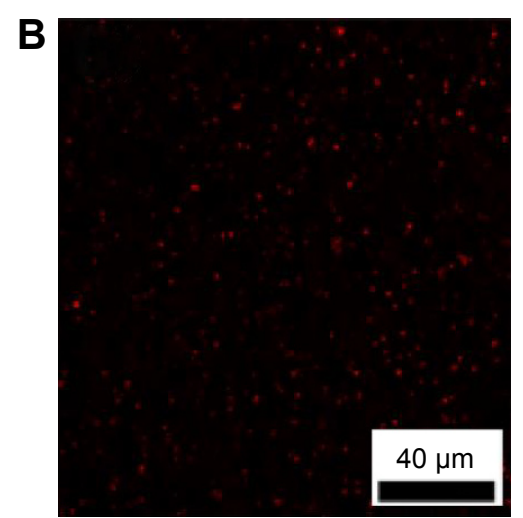

E

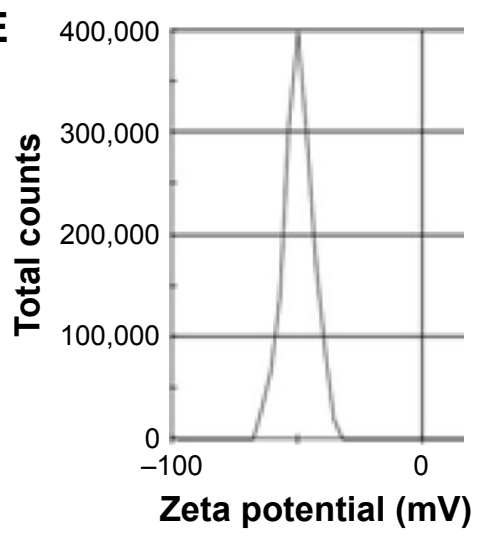

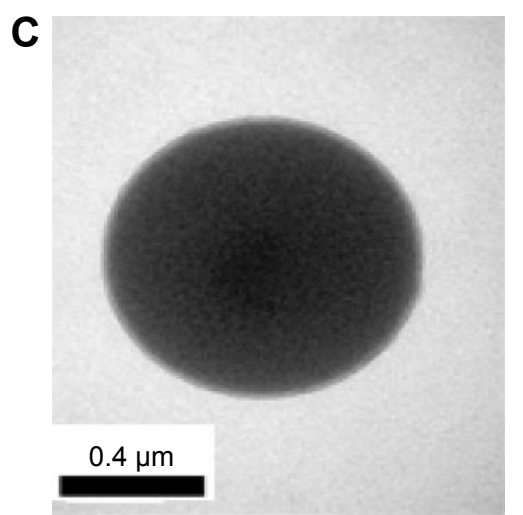

$\mathbf{F}$

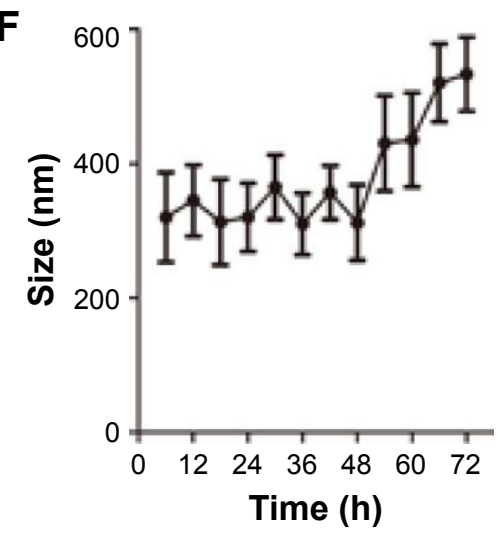

Figure 2 Characterization of nanodroplets.

Notes: (A) Optical microscopy image of FA-NDs. (B) CLSM image of Dil-stained FA-NDs. (C) TEM image of FA-NDs. (D and E) Size distributions and zeta potential of FA-NDs. (F) Size distribution of FA-NDs at $4^{\circ} \mathrm{C}$ after long-term storage $(n=3)$.

Abbreviations: FA-ND, folate-targeted perfluoropentane nanodroplet; CLSM, confocal laser scanning microscopy; TEM, transmission electron microscope; Dil, I,I'dioctadecyl-3,3,3',3'-tetramethylindocarbocyanine perchlorate. 

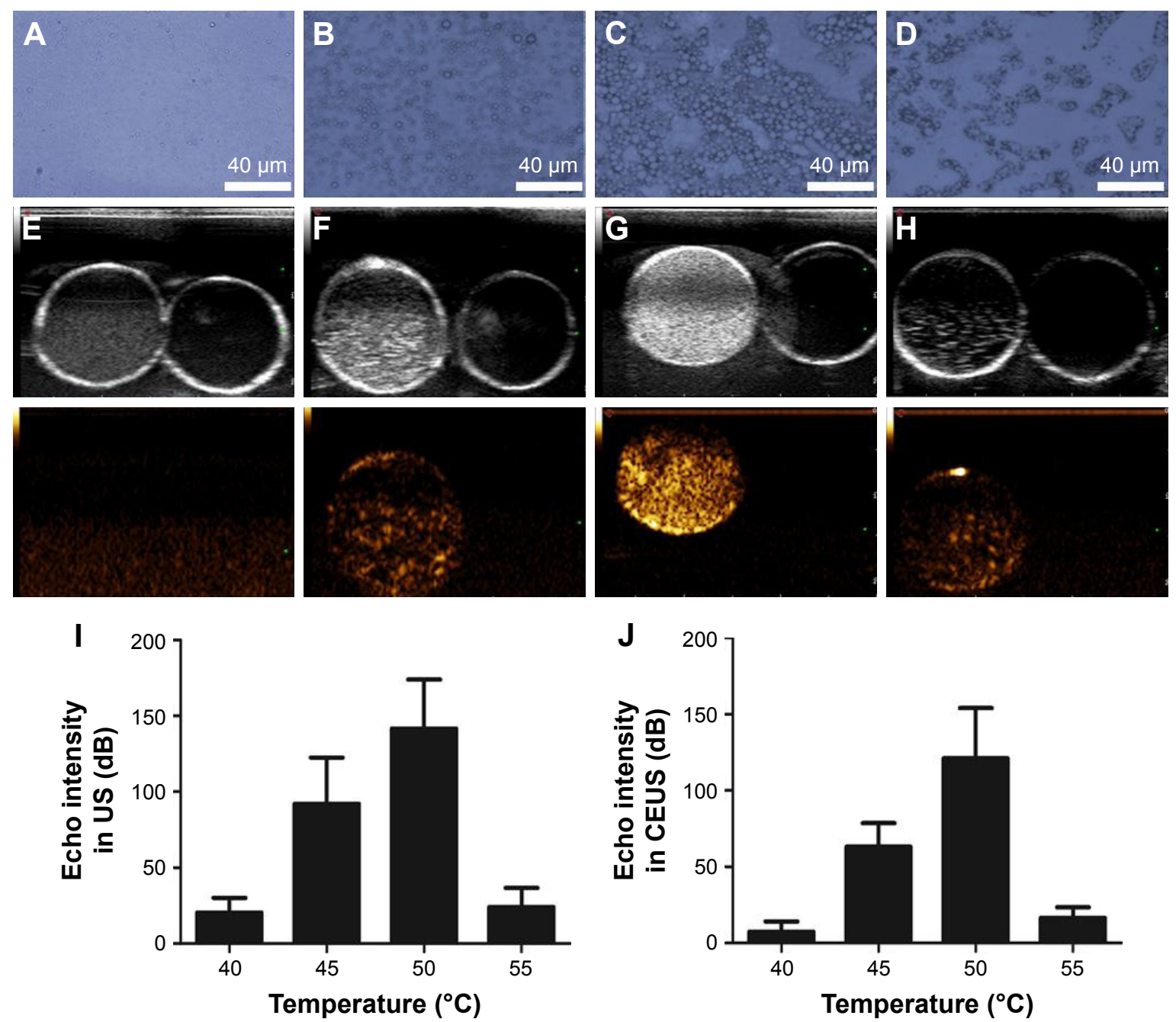

Figure 3 In vitro thermal evaporation of PFP nanoemulsions.

Notes: Optical microscopic images of FA-ND nanoemulsions heated to different temperatures $\left(40^{\circ} \mathrm{C}[\mathbf{A}], 45^{\circ} \mathrm{C}[\mathrm{B}], 50^{\circ} \mathrm{C}[\mathbf{C}], 55^{\circ} \mathrm{C}[\mathrm{D}]\right)$. US images of nanoemulsions in water suspensions of different temperatures $\left(40^{\circ} \mathrm{C}[\mathbf{E}], 45^{\circ} \mathrm{C}[\mathbf{F}], 50^{\circ} \mathrm{C}[\mathbf{G}], 55^{\circ} \mathrm{C}[\mathbf{H}]\right)$; the upper and lower panels show the B- and $\mathrm{CEUS}$ modes, respectively. Echo intensity in B-mode (I) and CEUS mode $(\mathbf{J})$ of nanoemulsion US imaging at different temperatures.

Abbreviations: PFP, perfluoropentane; FA-ND, folate-targeted perfluoropentane nanodroplet; US, ultrasound; CEUS, contrast-enhanced ultrasound.

In the in vitro ADV experiment with LIFU, there was no US enhancement in either mode when the LIFU acoustic intensity was $0.8 \mathrm{~W} / \mathrm{cm}^{2}$ (Figure $4 \mathrm{~A}$ and $\mathrm{E}$ ). When the intensity was increased to $1.6 \mathrm{~W} / \mathrm{cm}^{2}$, minimal US enhancement was observed (Figure 4B and F). Enhancement for both the imaging modes peaked at $2.4 \mathrm{~W} / \mathrm{cm}^{2}$ (Figure $4 \mathrm{C}$ and $\mathrm{G}$ ). Conversely, enhancement decreased substantially when LIFU acoustic intensity was increased to $3.2 \mathrm{~W} / \mathrm{cm}^{2}$ (Figure 4D and $\mathrm{H}$ ). The optical microscopic results of particles after LIFU sonication were consistent with that of US imaging; the amount of MBs peaked when LIFU acoustic intensity was $2.4 \mathrm{~W} / \mathrm{cm}^{2}$, and MBs decreased rapidly and enlarged at $3.2 \mathrm{~W} / \mathrm{cm}^{2}$ (Figure $4 \mathrm{I}-\mathrm{L}$ ). The quantitative results measured with US imaging analysis software revealed that when LIFU acoustic intensity was $2.4 \mathrm{~W} / \mathrm{cm}^{2}$, the echo intensities were highest in B-mode and CEUS mode (Figure 4M and N).

\section{Targeting efficiency of FA-targeted nanoemulsions}

Nanodroplet targeting efficiency was evaluated in vitro and in vivo. In order to accurately observe the targeting effect of nanodroplets to SKOV3 cells, CLSM was used and triple fluorescent labeling experiments (red fluorescence from DiI-labeled nanodroplets, green fluorescence from DiO-labeled cell membranes, and blue fluorescence from DAPI-labeled nuclei) was performed. As shown in Figure 5, numerous red dots representing FA-NDs were visible around the cell membrane and in the cytoplasm of SKOV3 cells in the targeted group, while minimal red signal was observed in cells in the non-targeted and antagonized groups.

To further validate the active targeting efficiency of FA-ND nanoemulsions in vivo, FLI was performed in samples from SKOV3 tumor-bearing nude mice. In targeted 


\section{$0.8 \mathrm{~W} / \mathrm{cm}^{2}$}
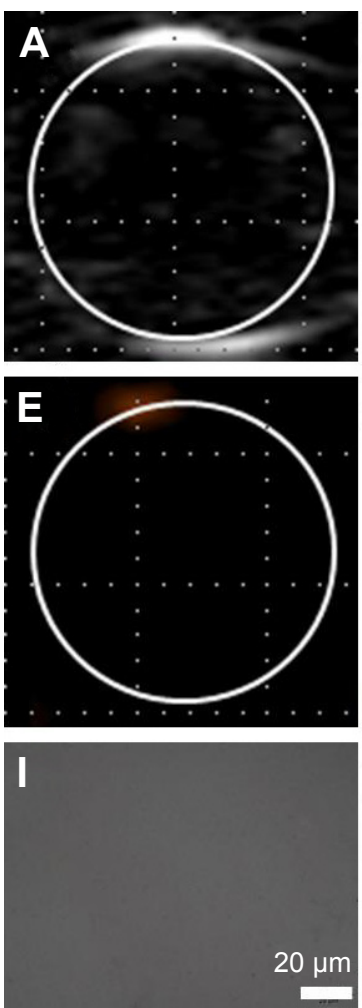

\section{M}
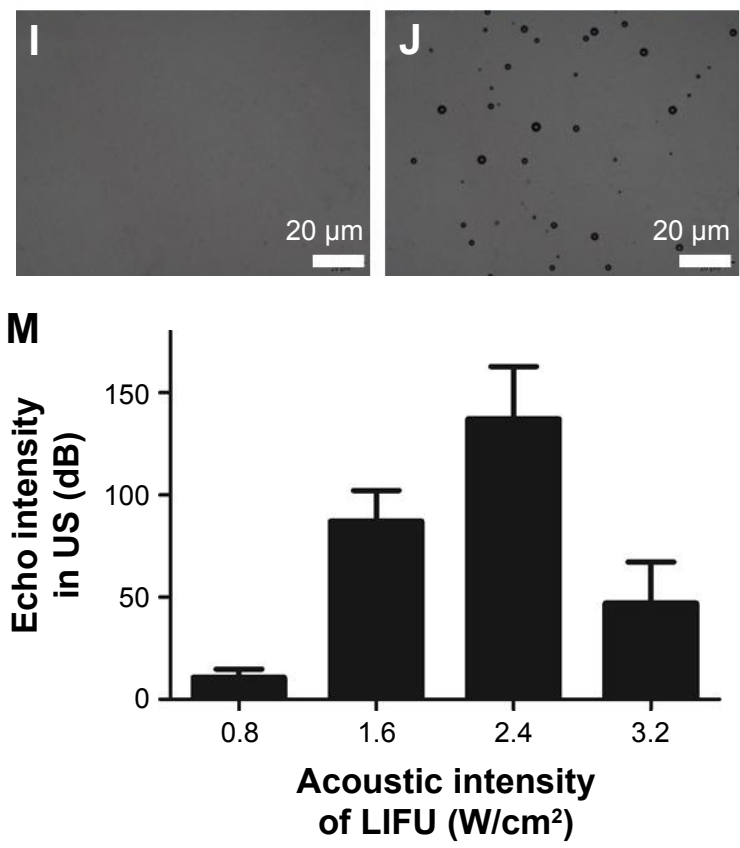

$20 \mu \mathrm{m}$
$2.4 \mathrm{~W} / \mathrm{cm}^{2}$
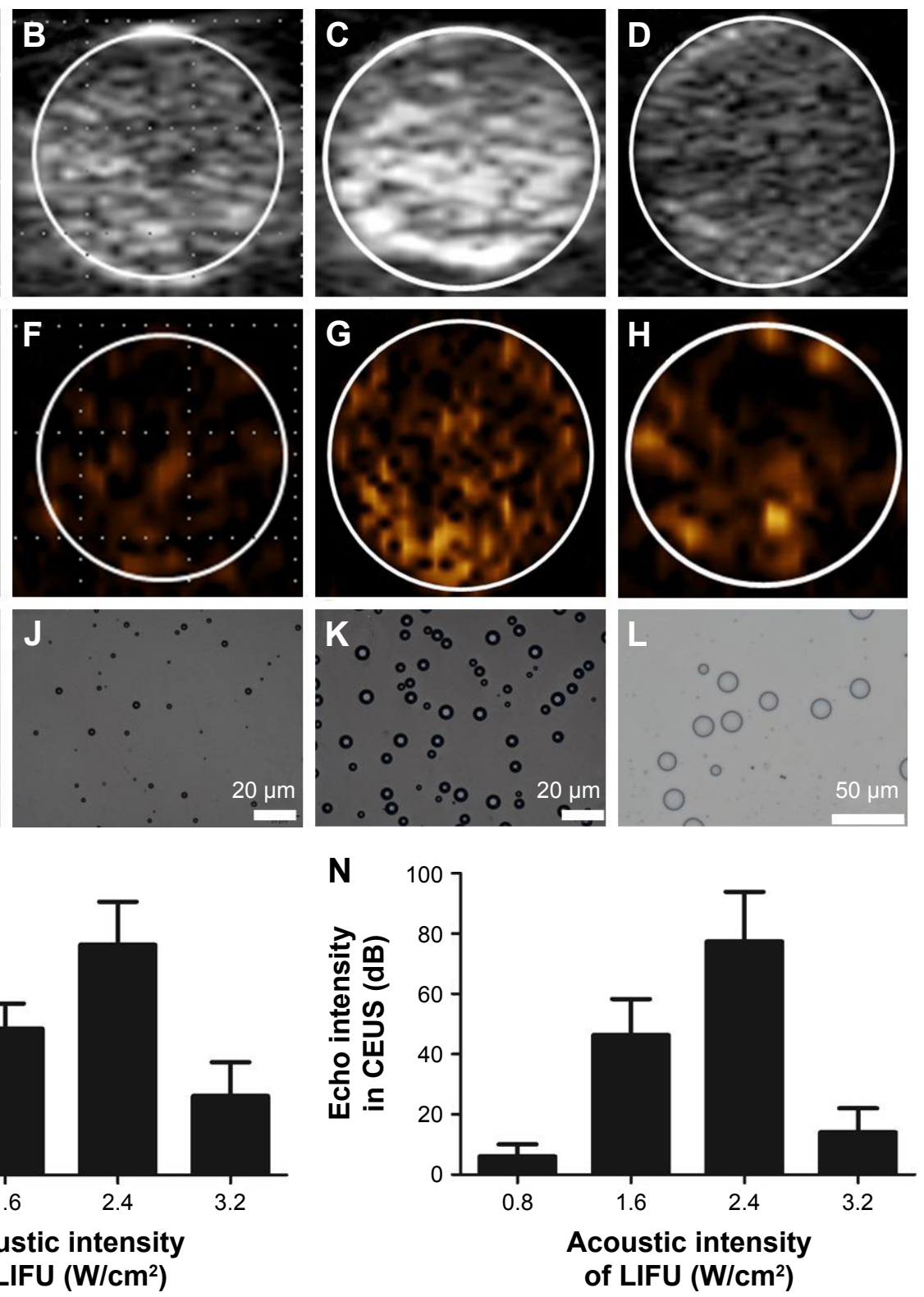

Figure 4 In vitro evaporation of PFP nanoemulsions through ADV.

Notes: US images of nanoemulsions (A-D: B-mode; E-H: CEUS mode). Optical microscopy showing particle shape (I-L) after 2-min sonication with different LIFU acoustic intensities. Echo intensities in B-mode (M) and CEUS mode $(\mathbf{N})$ of nanoemulsions after LIFU sonication.

Abbreviations: PFP, perfluoropentane; ADV, acoustic droplet vaporization; US, ultrasound; CEUS, contrast-enhanced ultrasound; LIFU, low-intensity focused ultrasound.

group, red fluorescence was seen in tumor area $0.5-12 \mathrm{~h}$ after the injection of FA-ND nanoemulsions dyed with $\mathrm{DiR}$, and the fluorescence intensity and range peaked at $1 \mathrm{~h}$ (Figure 6A-D). In the non-targeted group, there was nearly no red fluorescence in the tumor area following the injection of non-ND nanoemulsions (Figure 6E-H). Ex vivo FLI at $12 \mathrm{~h}$ postinjection showed that tumor nodes in the targeted group showed red fluorescence, but those in the non-targeted group did not (Figure 6I and J). The tumor-to-background fluorescence intensity ratios at $0.5,1,6$, and $12 \mathrm{~h}$ postinjection were significantly different between the targeted and non-targeted groups $(P<0.01)$ (Figure 6K). The ex vivo distribution of fluorescence intensity in mouse tissues at $12 \mathrm{~h}$ postinjection revealed that the tumors of the targeted group showed strong fluorescence intensities except in the liver and spleen, and fluorescence intensities of tumors in the targeted group were much stronger than those in the non-targeted group $(P<0.05)$ (Figure 6L). Examination of ultrathin tumor section showed that the targeted group contained many red dots representing FA-NDs (Figure 6M-P), and the highest number was noted at $1 \mathrm{~h}$. Virtually no red dots were found in tumor sections from the non-targeted group (Figure 6Q-T). 

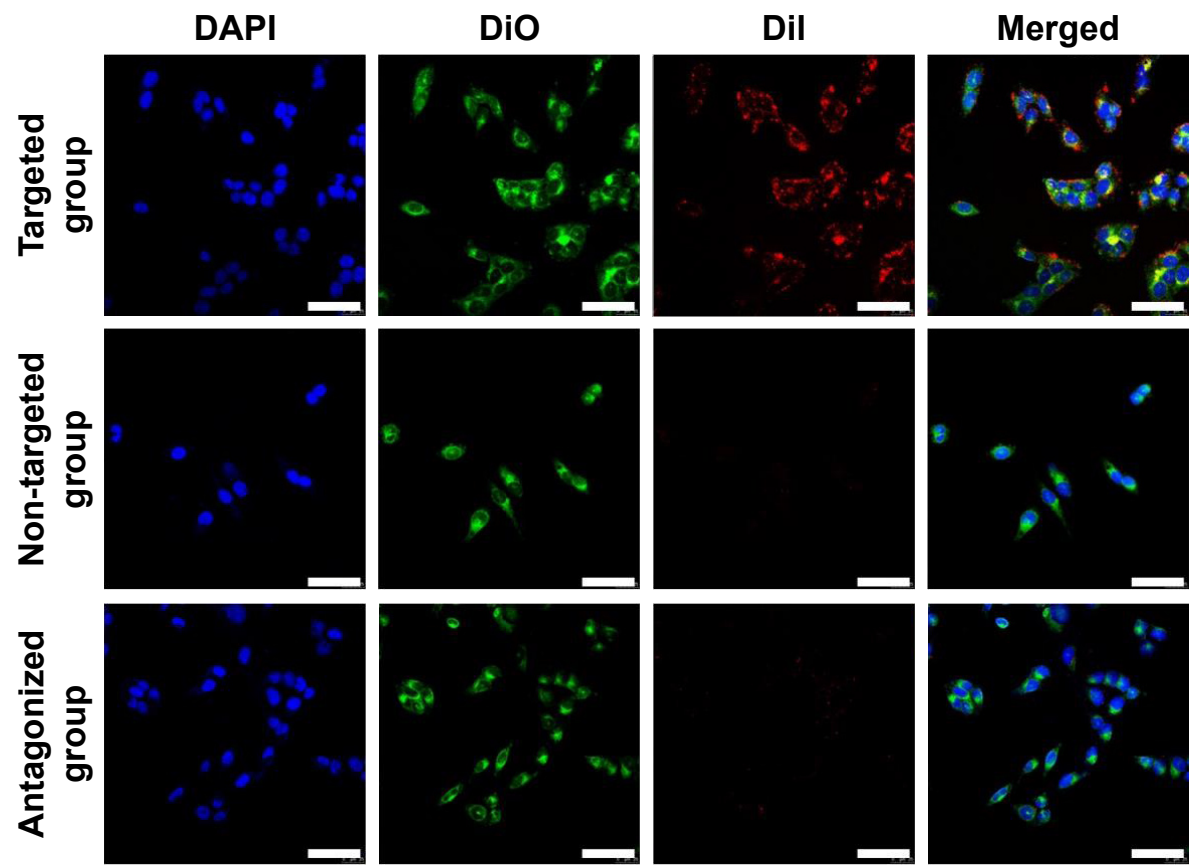

Figure 5 In vitro FA-NDs targeting efficiency.

Notes: CLSM images of SKOV3 cells after incubation with FA-NDs or non-NDs. In comparison, SKOV3 cells were first incubated with excess free FA and then FA-ND nanoemulsions under the same conditions (scale bar: $50 \mu \mathrm{m}$ ). Blue, DAPI-stained nuclei; green, DiO-labeled plasmalemma; red, Dil-labeled nanodroplets.

Abbreviations: FA-ND, folate-targeted perfluoropentane nanodroplet; CLSM, confocal laser scanning microscopy; DAPI, 4',6-diamidino-2-phenylindole; DiO, 3,3'dioctadecyloxacarbocyanine perchlorate; Dil, I, I'-dioctadecyl-3,3,3',3'-tetramethylindocarbocyanine perchlorate.

\section{In vivo $A D V$ observation}

When the acoustic intensity was $<3.2 \mathrm{~W} / \mathrm{cm}^{2}$, no US contrast enhancement was found in the tumor region, which indicated insufficient ADV induction of FA-NDs in the tumor (data not shown). With a fixed LIFU acoustic intensity of $3.2 \mathrm{~W} / \mathrm{cm}^{2}$, US enhancement was visible when the duration was 2 min (data not shown), but longer duration did not elevate US enhancement. Therefore, subsequent in vivo ADV experiments were performed with $3.2 \mathrm{~W} / \mathrm{cm}^{2}$ of acoustic intensity for $2 \mathrm{~min}$.

Before the injection of nanoemulsion, SKOV3 tumor nodules showed regular low echo tissues. After intravenous injection of FA-ND nanoemulsions followed by LIFU sonication, both B-mode and CEUS-mode imaging enhancement of tumor nodules was observed in the tumor region (Figure 7A), indicating that adequate nanodroplets were accumulated around tumor cells and underwent phase transition under LIFU sonication. The range of CEUS enhancement in the tumor was in agreement with the area of the LIFU focal spot. Although no obvious US enhancement was observed in the whole tumor nodules after the injection of non-ND nanoemulsions and LIFU sonication (Figure 7A), resulting from no nanodroplets being accumulated around tumor cells. No obvious enhancement was observed either in the tumor after the injection of FA-ND nanoemulsions without LIFU sonication (Figure 7A), which indirectly demonstrated that in the mice injected with FA-NDs followed by LIFU sonication, it was not physiological temperature but LIFU sonication that induced ADV phenomenon. In the mice injected with FA-ND nanoemulsions followed by LIFU sonication, the quantitative value of echo intensity increase in B-mode and CEUS mode were substantially higher than that of the other mice (Figure 7B and C).

\section{Discussion}

The developed phase changeable FA-NDs in our study exhibited favorable characteristics for US molecular imaging in tumor, including a relative small size, good stability, and controllable vaporization. The average diameter of FA-NDs was $\sim 321 \mathrm{~nm}$, indicating that most of the FA-NDs were in the reported particle size range ( $200 \mathrm{~nm}$ to $1.2 \mu \mathrm{m}$ ) eligible for EPR effect ${ }^{25}$ and for targeted imaging and therapy. The small size offered good penetrability and stability for FANDs, which was beneficial to extravascular US molecular imaging. However, the threshold of phase transition for small nanodroplets may be simultaneously increased and so a relatively higher US energy was required to induce ADV for US enhancement, which would increase the risk to damage the surrounding normal tissue. This study chose PFP with a low BP $\left(29^{\circ} \mathrm{C}\right)$ as the core of FA-NDs to decrease 
their threshold of phase transition. As known already, the BP of PFP is only $29^{\circ} \mathrm{C}$, which indicated that nanodroplets encapsulating PFP may be converted into gas bubbles at physical temperature of $37^{\circ} \mathrm{C}$ due to the phase transition.

However, the prepared FA-NDs in this study could remain stable even at $40^{\circ} \mathrm{C}$, which may be attributed to their small size and elastic shells. After being encapsulated into a phospholipid shell to formulate a nano-sized droplet, the BP of FA-NDs can be elevated substantially due to the high Laplace pressure. ${ }^{26}$ The results of in vitro thermal evaporation
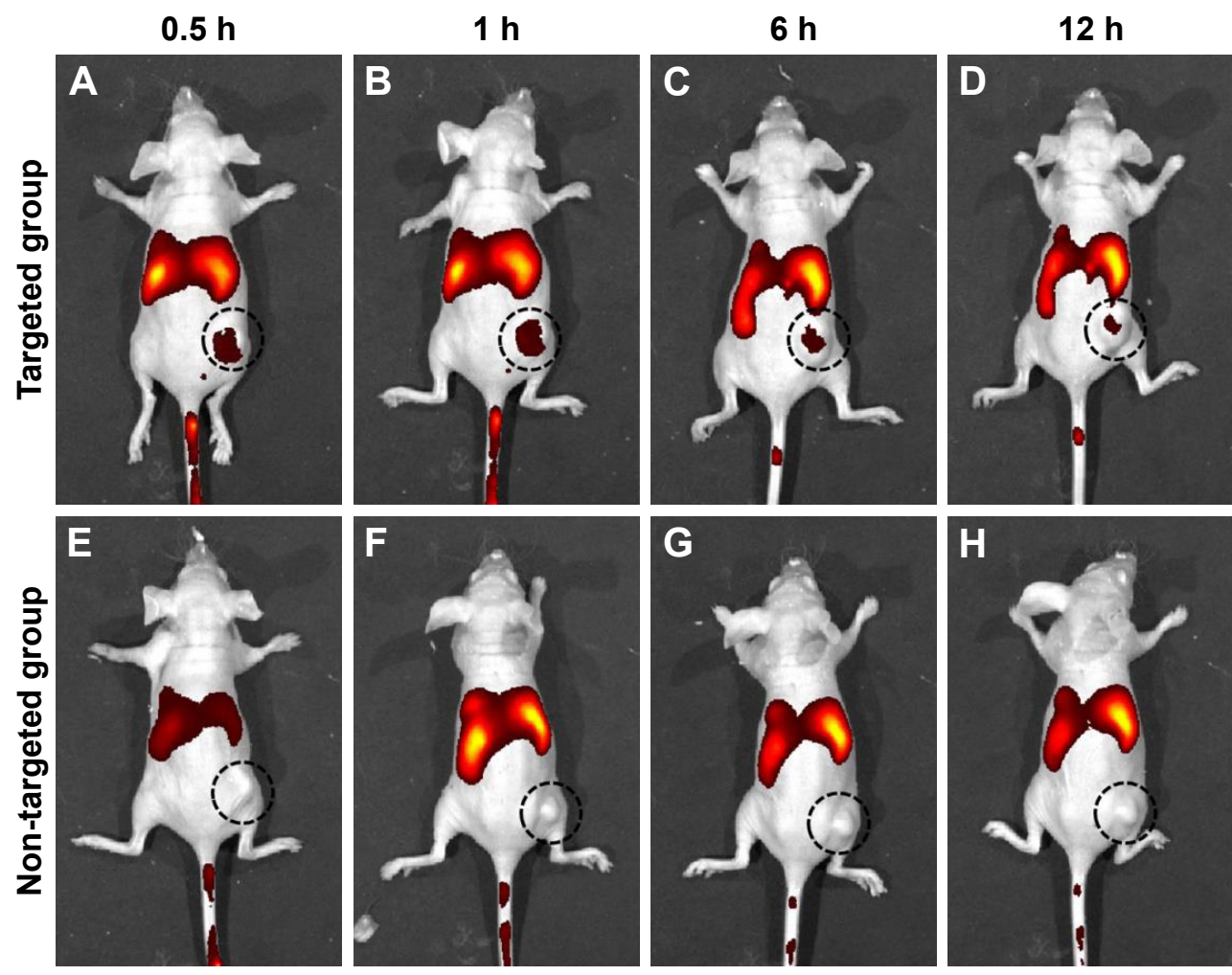

Epi-

fluorescence

I
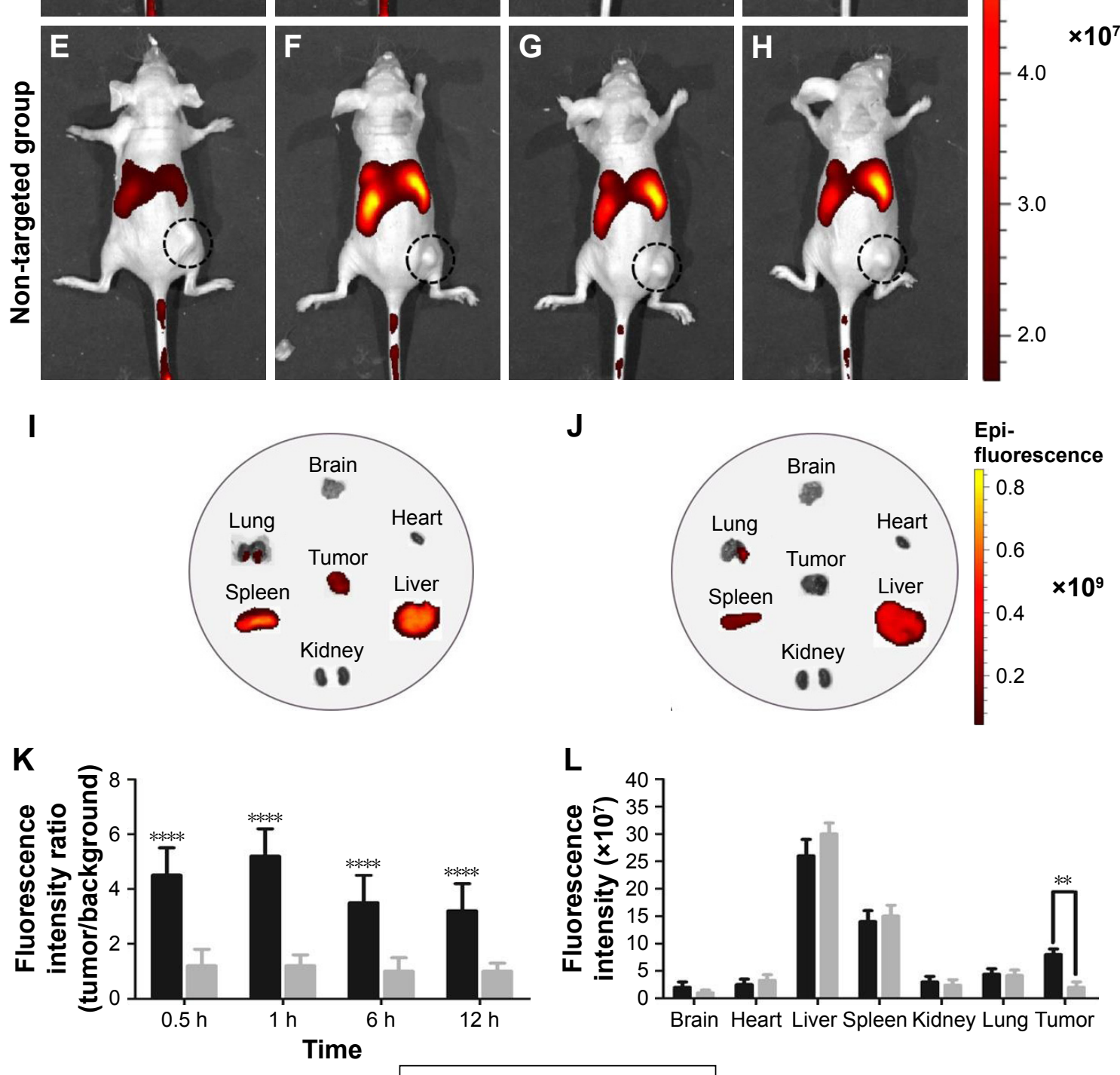

Figure 6 (Continued) 

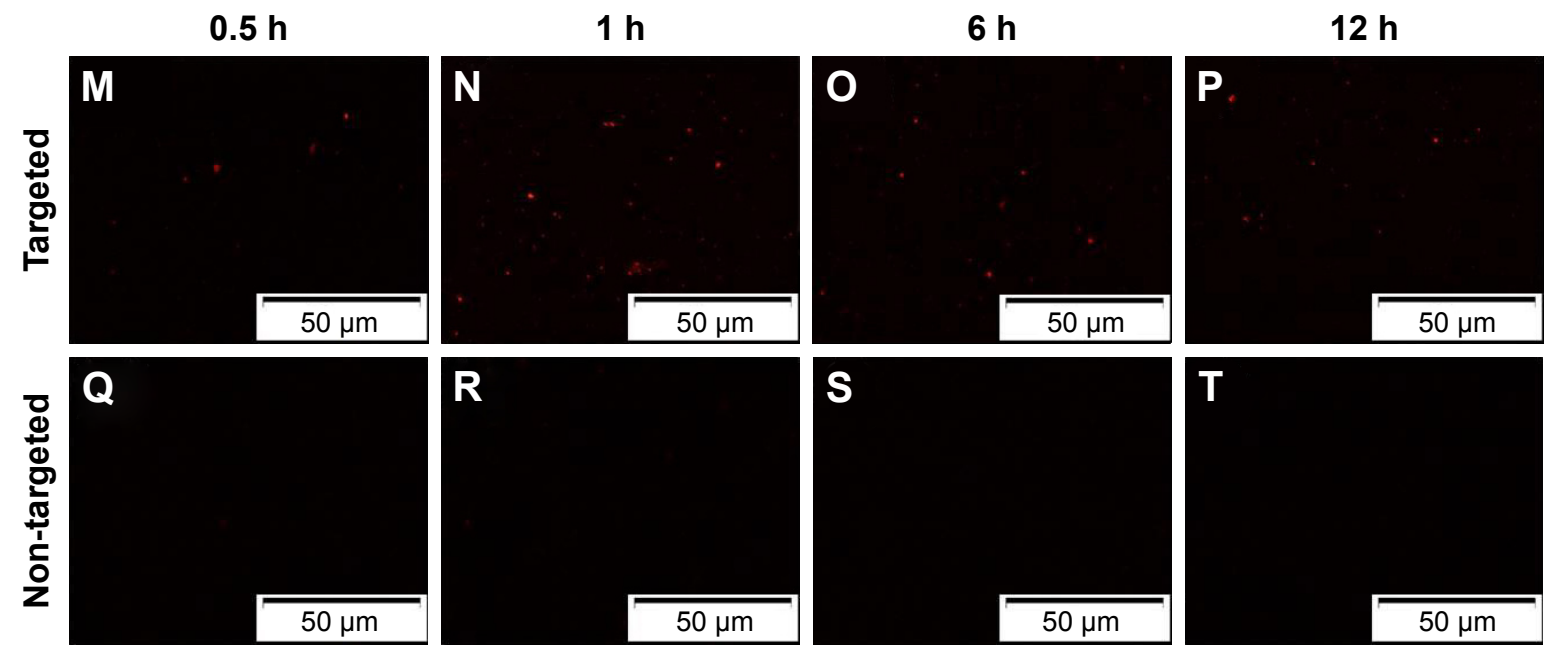

Figure 6 FA-NDs targeting efficiency using FLI 0.5, I, 6, and $12 \mathrm{~h}$ after the injection of nanoemulsion.

Notes: Black dotted circles mark tumor nodules. FLI image of a mouse at different time points (0.5, I, 6, and I2 h) post-injection of (A-D) FA-NDs and (E-H) non-NDs nanoemulsions dyed with DiR. Ex vivo FLls of main organs and tumors $12 \mathrm{~h}$ after the injection of (I) FA-ND and (J) non-ND nanoemulsions. (K) In vivo tumor/background fluorescence intensity ratio measured at time points of $0.5, \mathrm{I}, 6$, and $12 \mathrm{~h}$ after intravenous administration of FA-NDs or non-NDs nanoemulsions ( $* * * * P<0.0 \mathrm{I}$ vs nontargeted group). (L) Mean fluorescence intensities of main organs and tumors $12 \mathrm{~h}$ after the administration of FA-ND and non-ND nanoemulsions (**P $<0.05$ ). FLls in ultrathin section of tumor tissues at different time points $(0.5, \mathrm{l}, 6$, and $12 \mathrm{~h})$ post-injection of (M-P) FA-ND and (Q-T) non-ND nanoemulsions dyed with Dil.

Abbreviations: FA-ND, folate-targeted perfluoropentane nanodroplet; FLI, fluorescence imaging; Dil, I, I'-dioctadecyl-3,3,3',3'-tetramethylindocarbocyanine perchlorate.

experiment indicated that the threshold of vaporization temperature could be $45^{\circ} \mathrm{C}$ and the phase transition reached the peak between $45^{\circ} \mathrm{C}$ and $50^{\circ} \mathrm{C}$. Obviously, the stability of FA-NDs at physiological temperature was helpful for the in vivo application. Moreover, DSPE-PEG (2000) was further used to compose nanodroplets to prolong lifespan in vivo, ${ }^{27}$ which could further increase their circulation time and local accumulation in tumor tissue.

The common US molecular probes were produced by attaching monoclonal antibodies to the MB shell surface, whereas the large protein molecules with large diameters restrict them to freely leave the vasculature and act as real cell targeting agent. In contrast, folate is a naturally occurring small molecules with high stability and non-immunogenic property, ${ }^{20,21}$ which is more favorable than the monoclonal antibodies. Folate shows high affinity with FA receptor (FR), which is frequently overexpressed in most malignant tumors, ${ }^{20,21,28}$ including ovarian cancer, endometrial cancer, breast cancer, colon cancer nasopharyngeal cancer, and so on. In this study, the folate-modified FA-NDs showed high binding affinity to SKOV3 tumor cells as shown by the targeting efficiency experiments in vitro and in vivo. In the CLSM experiment in vitro, many FA-NDs were found around SKOV3 tumor cells in targeted group while few around SKOV3 tumor cells in the antagonized group, demonstrating their specific binding ability of FA-NDs to SKOV3 tumor cells. As demonstrated by in vivo FLI, though many FA-NDs were retained in the liver and spleen because of the reticuloendothelial system (RES) uptake, a remarkable targeting accumulation in tumor area was simultaneously found in the targeted group. Red fluorescence region was clearly found in tumor tissue in the FA-targeted group, while nearly no fluorescence in non-targeted group, suggesting that accumulation of nanodroplets in tumor tissue for ADV was mainly attributed to active targeting rather than passive targeting. ${ }^{29}$ The targeting efficiency of FA-NDs was further confirmed through ex vivo images and quantitative analysis. Meanwhile, the results from the ultrathin section also directly displayed the accumulation of FA-NDs in the tumor of the targeted group. Targeting distribution in tumor tissue of FA-NDs offer great promise for further US enhancement after nanodroplet ADV.

The in vitro and in vivo results demonstrated that phase transition of FA-NDs could be triggered and controlled by LIFU sonication. In in vivo ADV experiment, US enhancement was shown only in FA-NDs plus LIFU group, which indicated that adequate FA-NDs had accumulated in tumor tissue and ADV had been induced by LIFU sonication. Besides, a relatively higher acoustic intensity was required for ADV in vivo compared to that in vitro. This could be because that a large proportion of nanodroplets penetrated into tumor tissue and accumulated in local area had a smaller size, which required a relatively higher US energy to trigger ADV. While in vitro ADV experiment, phase transition would be initially generated in larger nanodroplets under LIFU sonication at a relatively lower intensity. It has been 
A
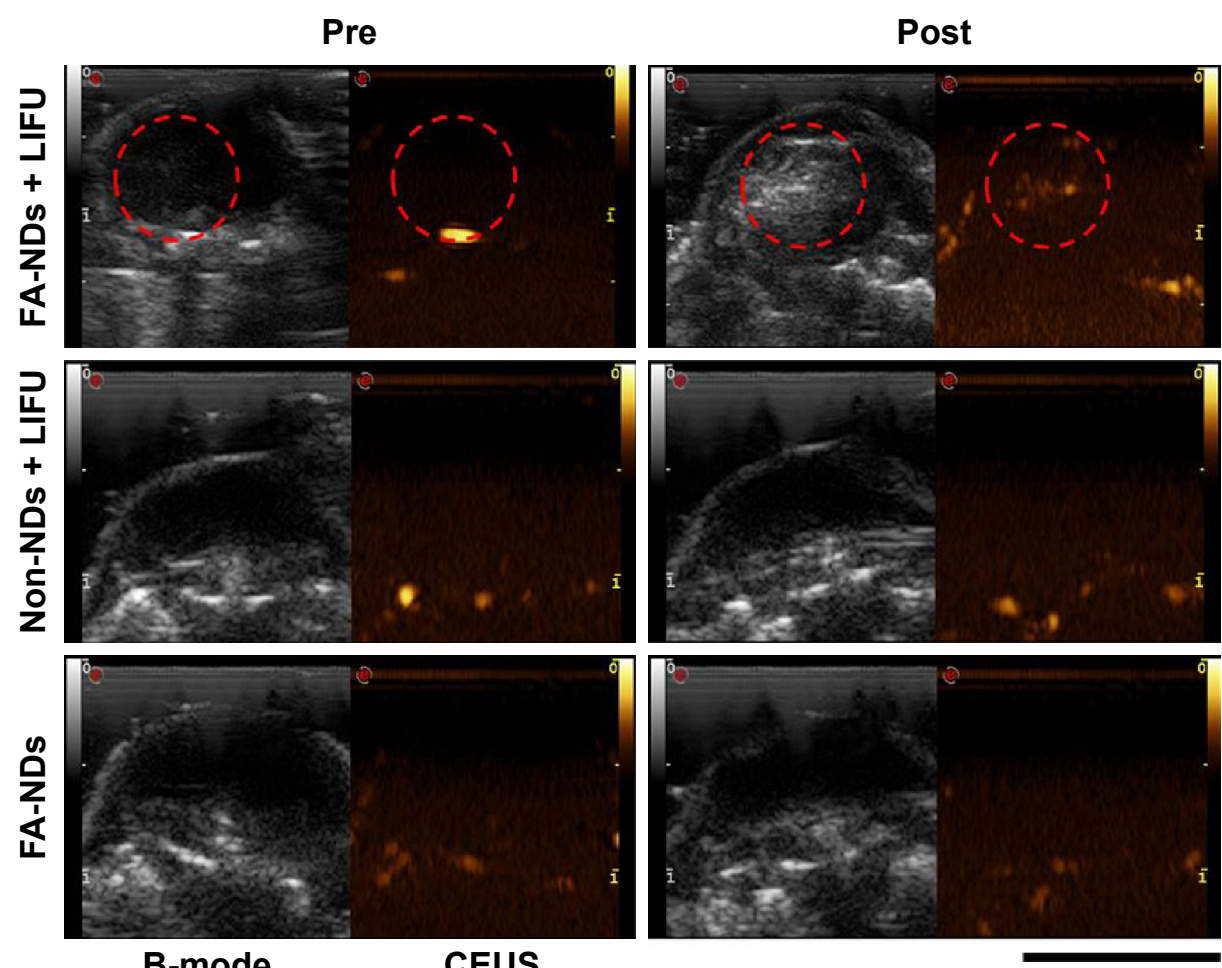

B-mode

CEUS

\section{B}

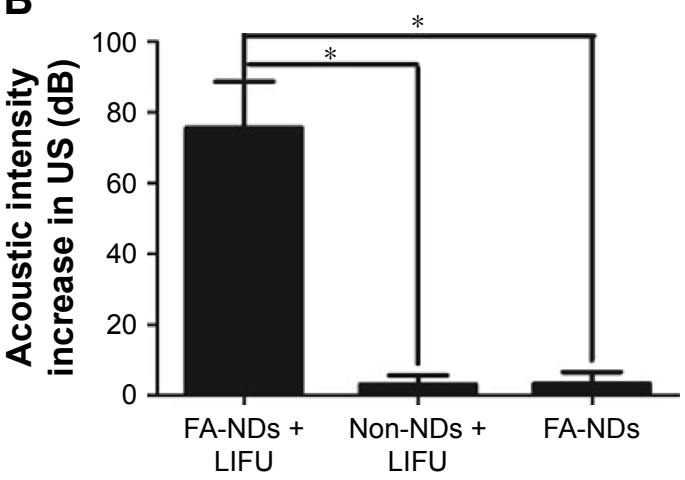

C

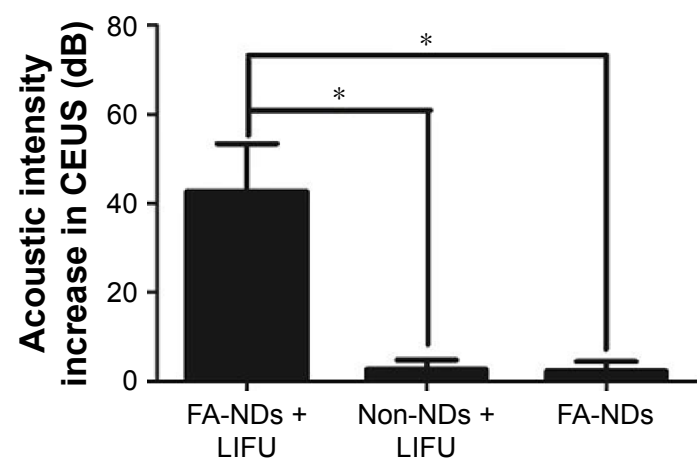

Figure 7 In vivo ADV with LIFU sonication.

Notes: (A) US images of mice tumors in B- and in CEUS modes before (pre) and after (post) different treatments (red dotted circles mark the area sonicated by LIFU exposure). Echo intensity increase in B-mode (B) and CEUS-mode $(\mathbf{C})$ of mice tumors $(* P<0.05)$.

Abbreviations: FA-NDs, folate-targeted perfluoropentane nanodroplets; ADV, acoustic droplet vaporization; LIFU, low-intensity focused ultrasound; US, ultrasound; CEUS, contrast-enhanced ultrasound.

reported that US energy required for droplet vaporization increased with the decrease in the droplet size. ${ }^{30}$

Compared to other methods that induced droplet-tobubble phase transition in nanodroplets, including heating and laser irradiation, US was generally recognized as the most accessible and effective stimulus. ${ }^{31}$ US generated many advantageous effects, including cavitation effect, mechanical effect, and heating effect, which efficiently promoted the phase-transition process of nanodroplets. ${ }^{32}$ HIFU has been reported to induce ADV in PFC nanodroplets in many studies, ${ }^{16,29,33}$ which can synergistically enhance the therapeutic efficiency of HIFU ablation on tumor. Clinically used
US imaging system has also been induced to activate droplet vaporization by increasing MI, but it could not accurately control the ADV area and it may be induced in the whole sonication scope ${ }^{34}$ Different from the previous work to trigger phase transition by HIFU, an LIFU instrument that produced lower intensity and focused acoustic energy was used to precisely induce ADV in tumor area as the encapsulated PFP core required a relatively low threshold of phase transition, which would reduce the nonspecific droplet-to-bubble transition around tumor and minimize the damage to healthy tissues surrounding the targeted site simultaneously. The focal zone of LIFU could be accurately located within the tumor region 
so that the phase transition of nanodroplets was controllable. Meanwhile, diagnostic ultrasonography system was instantly employed to scan the tumor area after LIFU sonication.

In conclusion, a type of folate targeted, phase changeable nanodroplets was developed as a novel molecular probe for tumor-targeted US imaging in this study, which showed high binding affinity and targeting efficiency to SKOV3 tumor cells through passive and active targeting effects, and low-intensity US sonication could precisely activate ADV effect in FA-NDs accumulating in tumor tissue. Finally, the US molecular imaging for tumor was achieved by the combination of FA-NDs and LIFU sonication through the ADV effect in this study. In addition, FA-NDs possess the potential to be used as drug carriers for tumor-targeted therapy in future study.

\section{Acknowledgments}

The authors are grateful to Jinshun $\mathrm{Xu}, \mathrm{PhD}$, for assistance in US imaging. This work was financially supported by the National Natural Science Foundation of the People's Republic of China (Grant Nos. 81371578, 81130025, 81227801, 81471675, and 31630026). Jianxin Liu and Tingting Shang are co-first authors who contributed equally to this work.

\section{Author contributions}

ZW, HR, PL, and ZD designed the experiments and guarantee the integrity of the study. JL, TS, YC, and LH wrote the main manuscript. JL and FW prepared the figures. JL, TS, and FW performed the experiments. JL, TS, YC, and JR performed literature research. All authors contributed toward data analysis, drafting and revising the paper and agree to be accountable for all aspects of the work.

\section{Disclosure}

The authors report no conflicts of interest in this work.

\section{References}

1. Weissleder R, Pittet MJ. Imaging in the era of molecular oncology. Nature. 2008;452(7187):580-589.

2. Li P, Zheng Y, Ran H, et al. Ultrasound triggered drug release from 10-hydroxycamptothecin-loaded phospholipid microbubbles for targeted tumor therapy in mice. J Control Release. 2012;162(2):349-354.

3. Wu J, Leong-Poi H, Bin J, et al. Efficacy of contrast-enhanced US and magnetic microbubbles targeted to vascular cell adhesion molecule-1 for molecular imaging of atherosclerosis. Radiology. 2011; 260(2):463-471.

4. Zhang K, Chen H, Guo X, et al. Double-scattering/reflection in a single nanoparticle for intensified ultrasound imaging. Sci Rep. 2015;5:8766.

5. Zhao YZ, Zhang M, Wong HL, et al. Prevent diabetic cardiomyopathy in diabetic rats by combined therapy of aFGF-loaded nanoparticles and ultrasound-targeted microbubble destruction technique. J Control Release. 2016;223:11-21.

6. Kopechek JA, Carson AR, McTiernan CF, et al. Ultrasound targeted microbubble destruction-mediated delivery of a transcription factor decoy inhibits STAT3 signaling and tumor growth. Theranostics. 2015;5(12):1378-1387.
7. Mozafari M, Shimoda M, Urbanska AM, Laurent S. Ultrasound-targeted microbubble destruction: toward a new strategy for diabetes treatment. Drug Discov Today. 2016;21(4):540-543.

8. Hobbs SK, Monsky WL, Yuan F, et al. Regulation of transport pathways in tumor vessels: role of tumor type and microenvironment. Proc Natl Acad Sci U S A. 1998;95(8):4607-4612.

9. Wilson KE, Wang TY, Willmann JK. Acoustic and photoacoustic molecular imaging of cancer. J Nucl Med. 2013;54(11):1851-1854.

10. Pysz MA, Machtaler SB, Seeley ES, et al. Vascular endothelial growth factor receptor type 2-targeted contrast-enhanced US of pancreatic cancer neovasculature in a genetically engineered mouse model: potential for earlier detection. Radiology. 2015;274(3):790-799.

11. Liu X, Zhao J, Guo D, et al. Synthesis and evaluation of perfluorooctylbromide nanoparticles modified with a folate receptor for targeting ovarian cancer: in vitro and in vivo experiments. Int J Clin Exp Med. 2015;8(6):10122-10131.

12. Zha Z, Wang J, Zhang S, et al. Engineering of perfluorooctylbromide polypyrrole nano-/microcapsules for simultaneous contrast enhanced ultrasound imaging and photothermal treatment of cancer. Biomaterials. 2014;35(1):287-293.

13. Li H, Wang J, Wang P, et al. Phase-transition contrast nanocapsules triggered by low-intensity ultrasound. Chem Commun (Camb). 2014; 50(96):15163-15166.

14. Seo M, Williams R, Matsuura N. Size reduction of cosolvent-infused microbubbles to form acoustically responsive monodisperse perfluorocarbon nanodroplets. Lab Chip. 2015;15(17):3581-3590.

15. Rapoport N. Phase-shift, stimuli-responsive perfluorocarbon nanodroplets for drug delivery to cancer. Wiley Interdiscip Rev Nanomed Nanobiotechnol. 2012;4(5):492-510.

16. $\mathrm{Ma} \mathrm{M}, \mathrm{Xu} \mathrm{H}$, Chen $\mathrm{H}$, et al. A drug-perfluorocarbon nanoemulsion with an ultrathin silica coating for the synergistic effect of chemotherapy and ablation by high-intensity focused ultrasound. Adv Mater. 2014;26(43):7378-7385.

17. Zhang X, Zheng Y, Wang Z, et al. Methotrexate-loaded PLGA nanobubbles for ultrasound imaging and synergistic targeted therapy of residual tumor during HIFU ablation. Biomaterials. 2014;35(19): $5148-5161$

18. You Y, Wang Z, Ran H, et al. Nanoparticle-enhanced synergistic HIFU ablation and transarterial chemoembolization for efficient cancer therapy. Nanoscale. 2016;8(7):4324-4339.

19. Wang X, Chen H, Chen Y, et al. Perfluorohexane-encapsulated mesoporous silica nanocapsules as enhancement agents for highly efficient high intensity focused ultrasound (HIFU). Adv Mater. 2012;24(6): 785-791.

20. Fang C, Veiseh O, Kievit F, et al. Functionalization of iron oxide magnetic nanoparticles with targeting ligands: their physicochemical properties and in vivo behavior. Nanomedicine (Lond). 2010;5(9): 1357-1369.

21. Li YJ, Dong M, Kong FM, Zhou JP. Folate-decorated anticancer drug and magnetic nanoparticles encapsulated polymeric carrier for liver cancer therapeutics. Int J Pharm. 2015;489(1-2):83-90.

22. Ren J, Xu C, Zhou Z, et al. A novel ultrasound microbubble carrying gene and Tat peptide: preparation and characterization. Acad Radiol. 2009;16(12):1457-1465.

23. Gong Y, Wang Z, Dong G, et al. Low-intensity focused ultrasound mediated localized drug delivery for liver tumors in rabbits. Drug Deliv. 2016:23(7):2280-2289.

24. Kripfgans OD, Orifici CM, Carson PL, Ives KA, Eldevik OP, Fowlkes JB. Acoustic droplet vaporization for temporal and spatial control of tissue occlusion: a kidney study. IEEE Trans Ultrason Ferroelectr Freq Control. 2005;52(7):1101-1110.

25. Yuan F, Dellian M, Fukumura D, et al. Vascular permeability in a human tumor xenograft: molecular size dependence and cutoff size. Cancer Res. 1995;55(17):3752-3756.

26. Gao D, Xu M, Cao Z, et al. Ultrasound-triggered phase-transition cationic nanodroplets for enhanced gene delivery. ACS Appl Mater Interfaces. 2015;7(24):13524-13537. 
27. Zhuang Y, Ma Y, Wang C, et al. PEGylated cationic liposomes robustly augment vaccine-induced immune responses: Role of lymphatic trafficking and biodistribution. J Control Release. 2012;159(1):135-142.

28. Iwakiri S, Sonobe M, Nagai S, Hirata T, Wada H, Miyahara R. Expression status of folate receptor alpha is significantly correlated with prognosis in non-small-cell lung cancers. Ann Surg Oncol. 2008; 15(3):889-899.

29. Zhou Y, Wang Z, Chen Y, et al. Microbubbles from gas-generating perfluorohexane nanoemulsions for targeted temperature-sensitive ultrasonography and synergistic HIFU ablation of tumors. Adv Mater. 2013;25(30):4123-4130.

30. Sheeran PS, Streeter JE, Mullin LB, Matsunaga TO, Dayton PA. Toward ultrasound molecular imaging with phase-change contrast agents: an in vitro proof of principle. Ultrasound Med Biol. 2013;39(5):893-902.
31. Rapoport NY, Efros AL, Christensen DA, Kennedy AM, Nam KH. Microbubble generation in phase-shift nanoemulsions used as anticancer drug carriers. Bubble Sci Eng Technol. 2009;1(1-2):31-39.

32. Rapoport NY, Kennedy AM, Shea JE, Scaife CL, Nam KH. Controlled and targeted tumor chemotherapy by ultrasound-activated nanoemulsions/ microbubbles. J Control Release. 2009;138(3):268-276.

33. Kripfgans OD, Zhang M, Fabiilli ML, et al. Acceleration of ultrasound thermal therapy by patterned acoustic droplet vaporization. J Acoust Soc Am. 2014;135(1):537-544.

34. Chen WT, Kang ST, Lin JL, Wang CH, Chen RC, Yeh CK. Targeted tumor theranostics using folate-conjugated and camptothecin-loaded acoustic nanodroplets in a mouse xenograft model. Biomaterials. 2015;53:699-708
International Journal of Nanomedicine

\section{Publish your work in this journal}

The International Journal of Nanomedicine is an international, peerreviewed journal focusing on the application of nanotechnology in diagnostics, therapeutics, and drug delivery systems throughout the biomedical field. This journal is indexed on PubMed Central, MedLine, CAS, SciSearch $®$, Current Contents $\AA /$ Clinical Medicine,

\section{Dovepress}

Journal Citation Reports/Science Edition, EMBase, Scopus and the Elsevier Bibliographic databases. The manuscript management system is completely online and includes a very quick and fair peer-review system, which is all easy to use. Visit http://www.dovepress.com/ testimonials.php to read real quotes from published authors.

Submit your manuscript here: http://www.dovepress.com/international-journal-of-nanomedicine-journal 